\title{
Proposition méthodologique en ergotoxicologie pour révéler les expositions à des produits chimiques
}

Methodological proposal in ergotoxicology to highlight chemical exposures

Louis Galey, Nathalie Judon, Caroline Jolly, Fabienne Goutille, Sarah Morelot, Marion Albert, Olivier Lhospital, Patrick Martin, Catherine NoelSuberville, Pierrick Pasquereau, Agnès Aublet-Cuvelier, Brahim Mohammed-Brahim et Alain Garrigou

\section{(2) OpenEdition}

\section{Journals}

Édition électronique

URL : http://journals.openedition.org/activites/4103

DOI : $10.4000 /$ activites.4103

ISSN : 1765-2723

Éditeur

ARPACT - Association Recherches et Pratiques sur les ACTivités

Référence électronique

Louis Galey, Nathalie Judon, Caroline Jolly, Fabienne Goutille, Sarah Morelot, Marion Albert, Olivier Lhospital, Patrick Martin, Catherine Noel-Suberville, Pierrick Pasquereau, Agnès Aublet-Cuvelier, Brahim Mohammed-Brahim et Alain Garrigou, «Proposition méthodologique en ergotoxicologie pour révéler les expositions à des produits chimiques », Activités [En ligne], 16-1 | 2019, mis en ligne le 15 avril 2019, consulté le 09 août 2019. URL : http://journals.openedition.org/activites/4103 ; DOI : $10.4000 /$ activites.4103

Ce document a été généré automatiquement le 9 août 2019

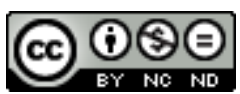

Activités est mis à disposition selon les termes de la licence Creative Commons Attribution - Pas d'Utilisation Commerciale - Pas de Modification 4.0 International. 


\section{Proposition méthodologique en ergotoxicologie pour révéler les expositions à des produits chimiques}

Methodological proposal in ergotoxicology to highlight chemical exposures

Louis Galey, Nathalie Judon, Caroline Jolly, Fabienne Goutille, Sarah Morelot, Marion Albert, Olivier Lhospital, Patrick Martin, Catherine NoelSuberville, Pierrick Pasquereau, Agnès Aublet-Cuvelier, Brahim Mohammed-Brahim et Alain Garrigou

\section{NOTE DE L'ÉDITEUR}

Article soumis le 27 juillet 2018, accepté le 13 février 2019

\section{NOTE DE L'AUTEUR}

\section{Conflit d'intérêts}

Les auteurs déclarent n'avoir aucun conflit d'intérêts associé à la publication de cet article. Son contenu, incluant les opinions et/ou conclusions exprimées, n'implique que les auteurs.

1 La recherche produisant ces résultats a été financée par la fondation ARC, l'INRS et la région Aquitaine. Les auteurs remercient également les entreprises et opérateurs qui ont accepté de participer à cette étude. 


\section{Introduction}

2 Cet article a pour objectif de poursuivre sur le plan méthodologique, la discussion proposée dans cette revue en 2009 par la publication d'« une approche critique du modèle dominant de prévention du risque chimique: L'apport de l'ergotoxicologie» par Mohammed-Brahim \& Garrigou. Cette publication avait permis de discuter le modèle dominant de prévention du risque chimique en milieu de travail, de ses apports comme de ses limites. Sur cette base avait été proposée une approche alternative: l'ergotoxicologie. L'ergotoxicologie est alors conçue comme une pratique particulière de l'ergonomie (Garrigou, 2011), qui vise à développer des modèles opérants (au sens de Wisner, 1972), des outils et des moyens de prévention efficaces pour gérer et prévenir les risques pour la santé des travailleurs exposés à des produits chimiques. On peut alors considérer que l'ergotoxicologie est un des domaines de spécialisation de l'ergonomie (Falzon, 2004, p. 18). Dans cette perspective, l'ergotoxicologie est ancrée profondément dans l'ergonomie, mais de par ses objectifs, elle mobilise des connaissances et des modèles issus de la toxicologie, de la médecine du travail et de la prévention. Sa pratique nécessite donc un engagement transdisciplinaire, en ne perdant pas de vue que ses racines se nourrissent de l'analyse de l'activité.

Dans la continuité de l'article de 2009, nous présentons ici des développements méthodologiques intégrant la technique de video exposure monitoring (VEM) (Rosén, Andersson, Walsh, Clark, Saämanen, Heinonen,... Pääkkönen, 2005) qui permet l'analyse conjointe de l'activité et des expositions. De cette façon, des mesures en temps réel sont associées à des vidéos de l'activité de travail. Cette méthodologie a été mise en œuvre dans trois entreprises (fabrication de peintures routières, fabrication de coques de bateaux et travaux de revêtement routier).

4 Le point de vue défendu est que les conditions d'exposition des travailleurs à ces dangers d'origine chimique constituent une énigme. Cette énigme n'est accessible aux opérateurs, à l'encadrement de l'entreprise et aux préventeurs que de manière fragmentée (Garrigou, Peeters, Jackson, Sagory, \& Carballeda, 2004). La méthodologie qui va être présentée cherche alors à mettre en visibilité et en discussion cette énigme. Les mesures et vidéos de situations de travail synchronisées par la technique de VEM constituent un support permettant de rassembler des données factuelles et objectives sur l'exposition (Judon, Hella, Pasquereau, \& Garrigou, 2015). La mise en discussions d'éléments objectifs et subjectifs issus des expositions devient alors possible avec l'opérateur observé, au sein de collectifs de travailleurs ou bien de collectifs incluant l'encadrement. L'usage de la VEM en ergotoxicologie produit ainsi des objets intermédiaires, décrits par Vinck (2009), au service de la prévention. De nouvelles perspectives pour les recherches et interventions en ergonomie et en hygiène industrielle, par l'usage de la VEM comme objet coopératif, apparaissent alors afin de mener collectivement des actions de transformation et de développement.

5 Cet article poursuit deux objectifs :

- Présenter la méthodologie développée en ergotoxicologie intégrant un outil de VEM ;

- Et présenter la nature des résultats produits, que ce soit au niveau de l'identification de situations d'exposition, de traitements statistiques des données ou bien de leur usage dans le cadre d'entretiens de confrontation afin de transformer les situations de travail. 
Ce texte s'adresse à des ergonomes, hygiénistes industriels, préventeurs ou médecins du travail, qui ont en commun d'être confrontés au cours de recherches, d'interventions ou bien de projets à des problématiques concernant des expositions de travailleurs à des produits chimiques.

\section{Apport de la VEM à l'hygiène industrielle}

7 Les premières utilisations de la VEM en entreprise par les hygiénistes industriels remontent au milieu des années 80 à une période où ces derniers rencontraient des difficultés dans l'interprétation des mesures réalisées (Rosén et al., 2005). Les résultats de mesurages obtenus à partir de courbes graphiques (enregistrement en temps réel) ne permettaient pas d'identifier et comprendre les éléments des situations de travail amenant à une exposition à des polluants atmosphériques sous forme de gaz, de vapeurs ou encore d'aérosols (Rosén et al., 2005). Plusieurs logiciels (PIMEX ${ }^{\oplus}$, VEM$^{\circledR}$, ELVis $^{\circledast}$, Captiv $\left.^{\oplus} . ..\right)$ ont vu le jour à cette période afin de synchroniser des enregistrements vidéo de situations de travail avec des enregistrements d'instruments de mesure en temps réel, de concentration de composés organiques volatils ou bien de particules (Martin, Brand, \& Servais, 1999).

8 Beurskens-Comuth, Verbist et Brouwer (2011) précisent que ce qui est important avec ce type de logiciel est de pouvoir «observer les variations des paramètres mesurés plutôt que de connaitre précisément la valeur obtenue» (p. 8, notre traduction). L'usage de la VEM pour développer les mesures d'hygiène sur le lieu de travail est fréquemment rapporté comme le potentiel pour générer de nouvelles idées afin de réduire l'exposition (Beurskens-Comuth, et al., 2011), renforcer la dynamique des acteurs de l'entreprise sur le sujet de l'exposition et améliorer la prise de conscience du risque (Rosén et al., 2005). Une hypothèse centrale de ces pratiques est que l'attitude des opérateurs peut être influencée positivement par la VEM. Ainsi, la présentation des résultats a souvent pour objectif de convaincre les opérateurs et influencer leur comportement plutôt que d'apprendre de leur expérience :

« [VEM] a fait ses preuves comme excellente méthode pour convaincre les employés de changer leur comportement et d'adhérer sur le long terme aux évolutions de process et pratiques de prévention» (Kuhl \& Dobernowsky, 2011, p. 79, notre traduction).

Dans le modèle classique de l'hygiène industrielle, ces données alimentent l'hypothèse selon laquelle l'opérateur serait souvent responsable de son exposition. Cet outil et les résultats qu'il produit cherchent alors à proposer des mesures correctives pour rectifier les mauvaises pratiques de l'opérateur générant ces expositions. La conséquence possible de ces usages de la VEM est de reproduire des pratiques de prévention où l'opérateur reste une cible de la prévention à atteindre et à convaincre, et non un acteur à part entière (Clot, 2010 ; Hale \& Borys, 2013 ; Mohammed-Brahim \& Garrigou, 2009 ; VidalGomel, 2017). L'absence de description des activités (au sens ergonomique du terme) et facteurs influençant l'activité des travailleurs est constatée dans cette littérature. Dans les approches en hygiène industrielle, les activités sont cibles de transformation au détriment des déterminants (techniques, organisationnels et humains) qui les influencent. L'analyse plurifactorielle de la situation d'exposition pouvant porter atteinte à la santé reste partielle par rapport aux pratiques décrites par Daniellou (1992), Garrigou et al. (2004), Mohammed-Brahim \& Garrigou (2009) et Vidal-Gomel (2017). De plus, la prise 
en compte des savoirs et représentations des opérateurs exposés n'est pas abordée dans les approches classiques en hygiène industrielle et en prévention, ce qui du point de vue de l'ergonomie reste une voie de développement des recherches et interventions portant sur les expositions professionnelles.

\section{Méthodologie d'usage de la VEM en ergotoxicologie}

Une méthodologie spécifique à l'intégration de l'outil VEM (voir Figure 1) a été élaborée en ergotoxicologie dans le cadre de différentes recherches-interventions. Nous présenterons plus particulièrement le travail mené dans trois entreprises entre 2013 et 2016. La demande générale des entreprises était d'avoir une meilleure compréhension de quand et comment les situations d'exposition à des produits chimiques potentiellement dangereux pour la santé se produisaient, afin d'identifier des actions d'amélioration de la prévention. En effet, les risques associés à ces expositions sont flous, différés et diffus (Mias, Legrand, Carricaburu, Féliu, \& Jamet, 2013). Les risques liés à ces substances sont difficiles à objectiver du fait de la complexité des situations d'exposition professionnelles, des fortes incertitudes scientifiques et sanitaires dont ils sont porteurs - dont la question récurrente des seuils et des faibles doses - et de la nature même des produits utilisés.

\subsection{Construction sociale des projets de recherche-intervention}

Dans le développement de cette méthodologie spécifique, il est important de préciser qu'un soin particulier a été porté à la construction sociale de l'intervention (Garrigou, Carballeda, \& Daniellou, 1998 ; Garrigou, Daniellou, Carballeda, \& Ruaud, 1995) pour chaque entreprise. Même si les interlocuteurs principaux étaient les préventeurs, chaque projet a été présenté aux différents acteurs de l'entreprise et au sein du comité d'hygiène, de sécurité et des conditions de travail. Dans chaque entreprise, des entretiens préalables ont été réalisés avec les opérateurs, l'encadrement, et les préventeurs pour : rassembler les documents de sécurité, recueillir des premières descriptions des situations d'exposition aux produits chimiques et comprendre comment les mesures de prévention avaient été élaborées. Par la suite, les entretiens de confrontation (voir partie résultats 4.3.) et la présentation de résultats avaient pour objectif de mobiliser différents acteurs de l'entreprise sur la thématique des expositions professionnelles. Ce travail de construction est important pour permettre aux acteurs de l'entreprise de comprendre les données d'observation produites et leur utilité, tout en développant un climat de confiance et une dynamique autour du projet. Dans cette perspective, l'ensemble des espaces de construction sociale a joué un rôle structurant dans le déroulement des projets. Ce travail de co-construction de l'intervention (Oddone, Re \& Brainte, 1981) a aussi conduit à sélectionner des situations de travail avec les opérateurs et l'encadrement pour des analyses approfondies articulant observations vidéo et réalisation de mesures en temps réel.

\subsection{Démarche méthodologique générale}

12 La démarche générale de la méthodologie est présentée dans la figure 1 suivante.

13 Elle comprend dans un premier temps le choix des paramètres physiologiques ou chimiques à mesurer. Ces paramètres vont alors dépendre de la nature de l'activité (en 
particulier la présence d'efforts physiques, qui ont un effet sur la fréquence cardiaque et respiratoire et donc sur l'exposition) et de la nature des produits chimiques (caractéristiques physico-chimiques de poudres, aérosols et gaz, leur dangerosité, etc.). Des instruments de mesure en temps réel adaptés à la fois à l'activité physiologique (cardio fréquencemètre, capteur de température cutanée, etc.) et à la nature de ces produits chimiques (instrument de mesure de composés organiques volatils ou bien de particules) sont choisis. Les instruments de mesure de concentration de produits chimiques ou de paramètres physiologiques doivent produire des enregistrements continus, afin d'être synchronisés aux enregistrements vidéo. Ces instruments et enregistrements vidéo sont ensuite mis en œuvre lors de l'observation des activités de travail.

Dans un deuxième temps, les données ainsi produites sont synchronisées à l'aide d'un outil de VEM. Un traitement est réalisé par l'intervenant pour identifier les situations d'exposition et analyser les activités de travail exposantes. Des extraits de situations d'exposition associant vidéo et mesure sont sélectionnés à partir des résultats d'analyse et des supports de VEM élaborés.

Dans un dernier temps, les extraits de situation d'exposition sont utilisés dans le cadre d'entretiens de confrontation et de restitutions avec les acteurs de l'entreprise dans l'objectif de renforcer la compréhension des situations d'exposition et la co-construction de transformations.

Figure 1 : Méthodologie générale d'usage de la VEM.

Figure 1: General methodology for VEM use

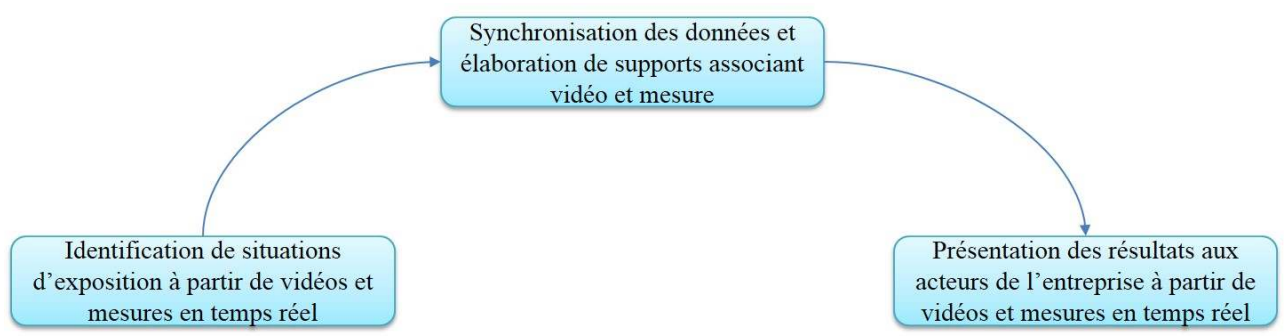

La méthode de construction et d'usage des supports de VEM est détaillée dans la partie 3.3.

\subsection{Méthode de construction et d'usage des supports de VEM}

17 Notre méthode de production et d'usage de supports issus de la technique de VEM utilise le logiciel Captiv ${ }^{\otimes}$, initialement développé par l'Institut National de Recherche et de Sécurité (INRS). 
Figure 2 : Méthode mise en œuvre dans les trois entreprises. Figure 2: Method of implementation within the three companies

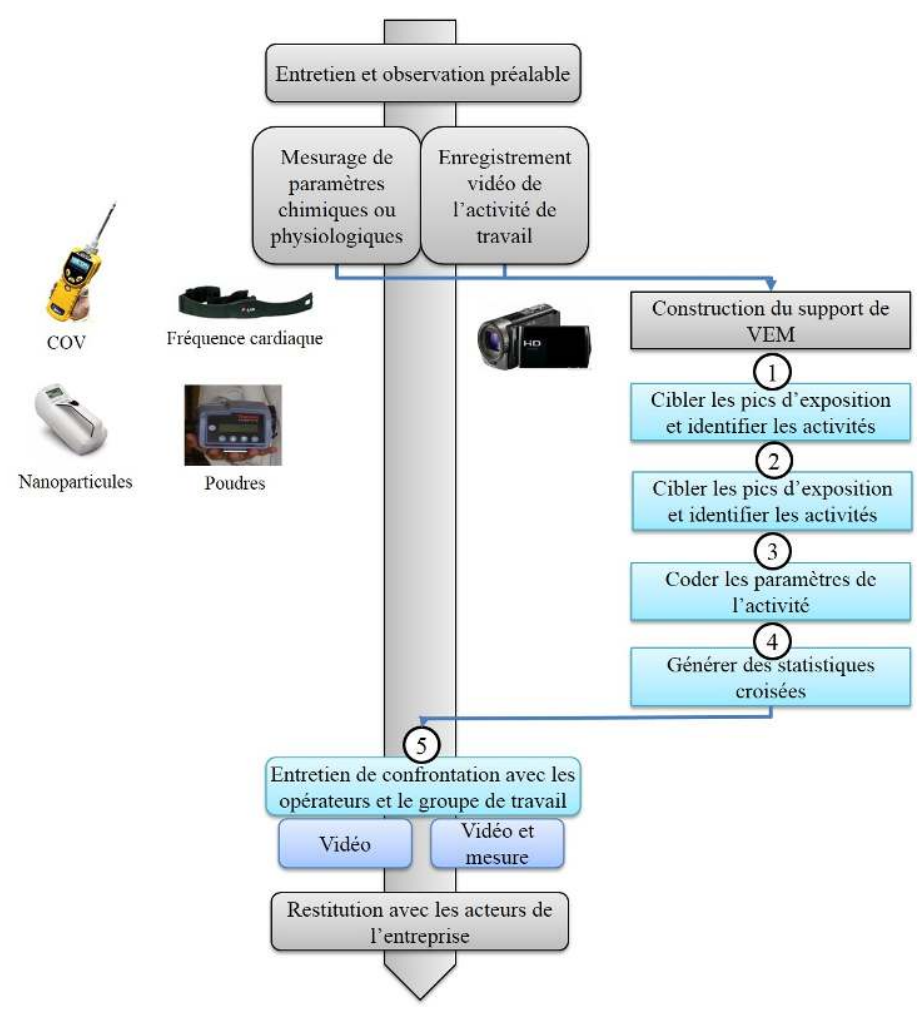

La méthode développée en ergotoxicologie pour l'utilisation de la VEM présentée en figure 2, comprend cinq étapes :

1. Étudier les variations des niveaux de concentration des polluants et en particulier les " pics», qui vont correspondre aux expositions significatives. De même, en ce qui concerne l'intensité physique du travail, des pics de fréquence cardiaque sont repérés. De même, le logiciel peut être utilisé pour repérer des situations avec une exposition plus faible ;

2. Caractériser les activités correspondant à ces pics, en positionnant le curseur sur le pic identifié, ce qui fait apparaître la vidéo correspondant à l'activité réalisée, grâce à la synchronisation de la vidéo et de la mesure ;

3. Coder l'activité afin de générer des statistiques sur les durées, fréquences et intensité de paramètres de l'activité. Il est ainsi possible de documenter les conditions d'exposition, à partir d'observables, définis par des paramètres qui influencent potentiellement l'exposition. Plusieurs «classes d'observables », comme par exemple " phases de l'activité », " postures », « produits chimiques manipulés », « usage des équipements de protection »...) peuvent être définies. Chacune d'entre elles va comporter des « observables exclusifs » (par exemple "porte le sac», "ouvre le sac», "vide le sac», "tasse le sac» pour la classe d'observable "phases de l'activité»). Ces observables sont choisis en fonction des hypothèses formulées sur les conditions de l'exposition. Ceci va permettre de réaliser un codage tel qu'il se pratique classiquement en analyse de l'activité (Kerguelen, 1997, 1998 et 2003). Par la suite, des chroniques d'activité représentant l'enchainement/interruption de ces classes d'observables et de ces observables sont ainsi produites;

4. Élaborer des statistiques sur les durées d'état des observables codés. L'outil de VEM Captiv ${ }^{\oplus}$, tout comme Actograph, permet de produire un premier niveau de traitement des données sur les durées cumulées et les fréquences d'apparition des différents observables. Pour ce qui concerne les données de mesures en temps réel, l'outil de VEM Captiv ${ }^{\circledast}$ permet de 
produire des données sur la durée pendant laquelle la concentration en produits chimiques est supérieure à un seuil défini par l'analyste ; cela donne une première analyse des niveaux d'exposition. Pour ce qui concerne les données de mesures physiologiques (fréquence cardiaque ou fréquence ventilatoire), des traitements statistiques sont proposés par l'outil de VEM Captiv ${ }^{\circledast}$ pour caractériser le niveau d'intensité physique. Un deuxième niveau de traitement plus élaboré est alors possible. Il permet de croiser des analyses portant sur les données de codage d'observables et des données de mesure. Par exemple, à partir de la classe d'observables phases de l'activité et des données de mesures en concentration de produits chimiques, il est possible d'identifier des niveaux de concentration et des durées d'exposition pour chaque phase d'activité. Dans la plupart des cas, l'exposition n'est pas homogène selon les phases de l'activité, ce qui conduit à identifier les phases de l'activité les plus exposantes au cours d'une journée de travail ;

5. Réaliser des entretiens de confrontation (Clot, 2008 ; Mollo \& Falzon, 2004) : dans un premier temps, l'entretien d'auto-confrontation se base sur la vidéo uniquement, l'image aidant la mise en mots de l'activité. Par les échanges qu'elle suscite avec le chercheur-intervenant, l'image permet à l'opérateur de se voir en activité et d'y découvrir des éléments nouveaux. Dans un deuxième temps, l'entretien d'auto-confrontation est conduit sur la base de présentation de séquences vidéo des mêmes situations de travail, mais cette fois-ci synchronisées aux données de mesurage. Les pics d'exposition préalablement identifiés par le chercheur et l'activité de travail correspondante présentés par le logiciel de VEM servent alors de support à la discussion portant sur les expositions. Les entretiens de confrontation sont réalisés d'abord avec le ou les opérateurs observés puis au sein de groupes de travail rassemblant les opérateurs et l'encadrement (responsable de production, directeur, agent de maîtrise, préventeur et médecin du travail). Ces entretiens peuvent ainsi être des autoconfrontations ou des confrontations collectives. Leur durée peut être comprise entre $1 \mathrm{~h} 30$ et $2 \mathrm{~h}$, ils sont enregistrés ou filmés avec l'accord des acteurs concernés. Ces entretiens doivent être réalisés dans les semaines qui suivent la réalisation des observations vidéo et des mesures pour s'assurer que les travailleurs puissent se remémorer les situations ;

6. L'ensemble des entretiens menés avec les opérateurs et l'encadrement est analysé en deux temps différents (Judon, 2017). Dans un premier temps, l'analyse consiste à extraire du discours les informations relatives à l'expérience/formation des opérateurs, à l'organisation des équipes, aux produits et substances utilisés, aux équipements de protection individuelle ainsi que toutes les évocations associées aux risques perçus de leur travail. Les verbatim sont ensuite analysés sous un angle plus précis où sont repérés plusieurs thèmes dans le discours des opérateurs (activités exposantes, déterminants de l'activité, perception et vécu de l'exposition, remise en question des pratiques de prévention notamment). Il s'agit d'obtenir des indicateurs qualitatifs permettant l'inférence de connaissances relatives aux conditions de production de verbatim à partir d'un ensemble de techniques d'analyse du discours (comme des procédures systématiques et objectives de description du contenu de ces verbatim), à la manière de ce que décrit Bardin (2011, p. 47).

19 Les restitutions avec les acteurs de l'entreprise en CHSCT ou en comité de pilotage sont constituées sur la base de l'ensemble des données recueilles et co-construites durant l'ensemble des étapes de la démarche. Ces phases de restitution permettent aux différents acteurs de dialoguer autour des supports de VEM appuyés des verbatim recueillis dans l'étape 5 si nécessaire. Notre méthodologie rapporte la réalisation de ces restitutions pour cohérence même si nos résultats ne les détaillent pas. 


\subsection{Particularités méthodologiques pour chaque entreprise}

Nous présentons ici les principaux choix méthodologiques adaptés aux problématiques rencontrées dans les trois entreprises :

Dans l'entreprise de peinture routière, le travail concerne une préparation de peinture routière sous forme de mélange de poudres. Ce mélange de poudres est issu de divers sacs de matière première manipulés par l'opérateur. Les poudres versées dans la trémie de chargement vont être mélangées automatiquement puis chargées par l'ensacheuse dans les sacs de poudres de peinture. Ainsi, il va s'agir pour l'opérateur de manutentionner les sacs de poudre de $25 \mathrm{~kg}$, les ouvrir, les vider, les fermer et les déplacer... Un instrument de mesure en temps réel de la concentration de particules de taille micrométrique et un instrument de mesure de la concentration en particules de taille nanométrique ont été choisis, de même qu'un cardio-fréquencemètre pour évaluer l'intensité physique. Le codage de l'activité sur l'ensemble des observations a porté sur les " phases de l'activité ».

Lors de travaux de revêtement routier, les opérateurs suivent un finisseur qui répartit le bitume chaud $\left(\simeq 180^{\circ} \mathrm{C}\right)$ sur la route. Les opérateurs doivent alors vérifier l'uniformité de la route, ajouter avec une pelle du bitume en cas de «trous » et « tirer » le bitume pour le répartir ou pour réaliser un joint entre deux bandes d'enrobé (mélange de bitume et de granulats utilisés pour recouvrir les chaussées). Ces activités se déroulent souvent dans des conditions de température extérieure élevée. Il a été choisi de mesurer la concentration en composés organiques volatils (COV) dégagés par le bitume chaud, la fréquence cardiaque pour évaluer l'intensité physique et la température cutanée pour caractériser le processus de thermorégulation. Le codage de l'activité sur l'ensemble des observations a porté sur les " phases de l'activité ».

Dans l'entreprise de fabrication de bateaux, l'analyse a porté sur le travail d'ébullage (écraser les bulles de vapeur de styrène qui peuvent être piégées entre les couches des matériaux composites) de coques de bateaux. Un instrument de mesure a été choisi pour mesurer la concentration en styrène présent sous forme gazeuse. Le capteur de fréquence cardiaque a été choisi pour évaluer l'intensité physique de l'activité. Le codage de l'activité a porté sur les « phases de l'activité » principalement.

4 Pour chaque entreprise, la nature des produits chimiques présents, le type d'instrument de mesure choisi, les stratégies d'observations ainsi que les entretiens et restitutions réalisés sont présentés dans le tableau 1. 
Tableau 1 : Description des terrains.

Table 1: Field description

\begin{tabular}{|c|c|c|c|}
\hline Entreprise & Polluant & Instrument de mesure & $\begin{array}{l}\text { Observation / mesurage approfondi et } \\
\text { entretiens de confrontation }\end{array}$ \\
\hline $\begin{array}{l}\text { 1. Fabrication } \\
\text { de peintures } \\
\text { routières }\end{array}$ & $\begin{array}{l}\text { Poudres } \\
\text { (résine, } \\
\text { silice, } \\
\text { dioxyde de } \\
\text { titane...) }\end{array}$ & 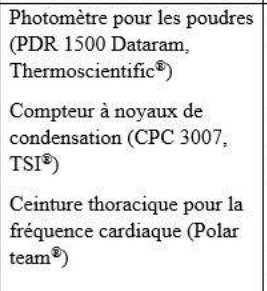 & $\begin{array}{l}4 \text { journées de travail avec deux équipes pour } \\
\text { des observations et mesurages approfondis } \\
1 \text { entretien de confrontation collective avec } \\
2 \text { opérateurs } \\
2 \text { entretiens de confrontation collective avec } \\
\text { le groupe de travail } \\
1 \text { restitution avec le comité d'hygiène, de } \\
\text { sécurité et des conditions de travail et le } \\
\text { comité de direction }\end{array}$ \\
\hline $\begin{array}{l}\text { 2. Travaux de } \\
\text { revêtement } \\
\text { routier }\end{array}$ & $\begin{array}{l}\text { Composés } \\
\text { organiques } \\
\text { volatils } \\
\text { (COV), } \\
\text { fumées }\end{array}$ & $\begin{array}{l}\text { Détecteur à photo ionisation } \\
\text { pour les COV (MiniRAE } \\
3000^{\S} \text { ) } \\
\text { Ceinture thoracique pour la } \\
\text { fréquence cardiaque (Polar } \\
\text { team }{ }^{2} \text { ) } \\
\text { Capteurs thermiques pour la } \\
\text { temperature cutanée } \\
\left(\text { Thermo Bouton, modèle : }_{\left.22 \mathrm{~L}, \text { Proges }^{\circledR}\right)}\right.\end{array}$ & $\begin{array}{l}2 \text { journées de travail avec une équipe pour } \\
\text { des observations et mesurages approfondis } \\
3 \text { entretiens d'auto confrontation avec les } \\
\text { opérateurs } \\
1 \text { entretien de confrontation collective avec } \\
\text { le groupe de travail } \\
1 \text { restitution incluant opérateurs de terrain, } \\
\text { chef d'équipe, préventeur, chef d'agence, } \\
\text { décideur de l'entreprise de construction } \\
\text { routière }\end{array}$ \\
\hline $\begin{array}{l}\text { 3. Fabrication } \\
\text { de bateau }\end{array}$ & $\begin{array}{l}\mathrm{COV} \\
\text { (styrène) }\end{array}$ & $\begin{array}{l}\text { Détecteur à photo ionisation } \\
\text { pour les COV (MiniRAE } \\
3000^{2} \text { ) } \\
\text { Ceinture thoracique pour la } \\
\text { fréquence cardiaque (Polar } \\
\text { team }^{2} \text { ) }\end{array}$ & $\begin{array}{l}4 \text { journées de travail avec } 4 \text { observations et } \\
\text { mesurages approfondis } \\
2 \text { entretiens d'auto confrontation avec les } \\
\text { opérateurs } \\
1 \text { entretien de confrontation collectif avec le } \\
\text { groupe de travail } \\
1 \text { restitution avec le comité d'hygiène, de } \\
\text { sécurité et des conditions de travail et le } \\
\text { comité de direction }\end{array}$ \\
\hline
\end{tabular}

\section{Principaux résultats}

Les résultats présentés ici sont issus de la mise en œuvre des 5 étapes de la méthode dans les trois entreprises (voir partie 3.3., Figure 2). Les situations d'exposition identifiées, certaines analyses statistiques réalisées et les résultats de l'usage des supports de VEM lors des entretiens de confrontation sont décrits successivement.

\subsection{Description des supports de VEM produits}

La vidéo affichée par le logiciel correspond à l'avancée du curseur sur les courbes représentant les données enregistrées par les instruments de mesure en temps réel à ce moment de la situation de travail. La chronique d'activité résultant du codage est affichée en parallèle des courbes issues des instruments de mesure à droite de l'écran.

Dans l'entreprise de peinture routière, nous observons une augmentation de la concentration en poussières et de la fréquence cardiaque lorsque l'opérateur tasse le sac (voir Figure 3); les valeurs instantanées, minimum et maximum, apparaissent à gauche des courbes. Cette action se déroule après que l'opérateur transporte et vide plusieurs sacs de matière première de $25 \mathrm{~kg}$ dans la trémie de chargement entraînant une augmentation de la fréquence cardiaque. Les sacs vides déposés par l'opérateur dans un sac-poubelle sont ensuite tassés. L'analyse montre que cette action chasse l'air des sacs et remet en suspension les poudres résiduelles des sacs. De plus, en raison des manutentions de sacs, l'opérateur a un niveau d'intensité physique lourd à très lourd (selon la grille de 
Meunier, Smolik, \& Knoché, 1994). En fonction de ces niveaux d'intensité physique, il est reconnu que le volume d'air inhalé par l'opérateur va augmenter (Horwat \& Meyer, 1998), favorisant la pénétration des polluants dans l'organisme.

Figure 3 : Capture d'écran du logiciel Captiv ${ }^{\circledR}$ illustrant une tâche de tassage de sac réalisée par un opérateur dans l'entreprise de peinture routière et une augmentation associée de l'exposition. Figure 3: Captiv ${ }^{\circledR}$ L7000-2014 software screenshot, describing a task performed by the worker and the associated increased of exposure in a road painting company
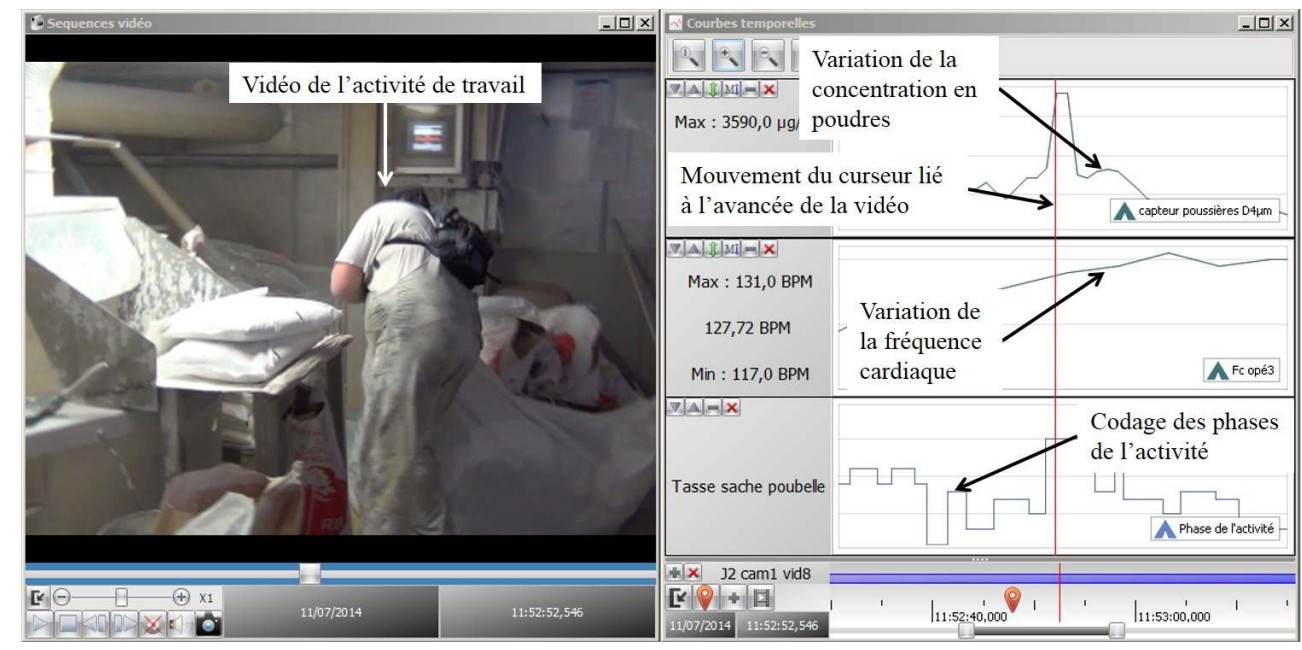

Lors de travaux de revêtement routier, la concentration en COV, la fréquence cardiaque et la température cutanée augmentent lorsque l'opérateur répand manuellement l'enrobé avec une pelle (voir Figure 4). Cette action se déroule après que de l'enrobé chaud $\left(\simeq 180^{\circ}\right.$ c) a été déversé sur la chaussée. L'analyse montre que l'opérateur est à proximité de l'enrobé et qu'il réalise un effort physique conséquent afin de pelleter l'enrobé à plusieurs reprises. L'intensité de l'effort physique peut correspondre dans certains cas à un niveau lourd à très lourd (grille de Meunier et al., 1994), ce qui se traduit aussi par une augmentation de la fréquence ventilatoire et donc par une augmentation du niveau d'inhalation de vapeurs de bitume. Des élévations de la température cutanée du bras et de la jambe à près de $39^{\circ} \mathrm{C}$ sont identifiées, ce qui atteste de contraintes physiologiques liées à la thermorégulation. Pour organiser son refroidissement, l'organisme humain doit envoyer le sang à la surface de la peau afin de permettre à la chaleur de sortir du corps. Les vaisseaux périphériques se dilatent au niveau cutané, permettant ainsi l'évacuation de la chaleur vers le milieu ambiant et favorisant les échanges avec l'extérieur. Cette vasodilatation entraîne alors une perméabilité plus grande de la peau aux substances exogènes et accroît le risque d'absorption cutanée, surtout lorsque le débit sanguin lié à l'effort est important (Monod \& Pottier, 1981). Il est donc probable que la quantité de substance exogène qui traverse potentiellement la barrière cutanée soit d'autant plus élevée que l'effort est intense.

La température extérieure moyenne sur cette journée d'observation était de $29^{\circ} \mathrm{C}$. 
Figure 4 : Capture d'écran du logiciel Captiv ${ }^{\circledR}$ illustrant une tâche réalisée par un opérateur dans l'entreprise de travaux publics et les variations associées de l'exposition.

Figure 4 : Captiv ${ }^{\circledR}$ L3000-2013 software screenshot, describing a task performed by the worker and the associated increased of exposure in a roadworks company

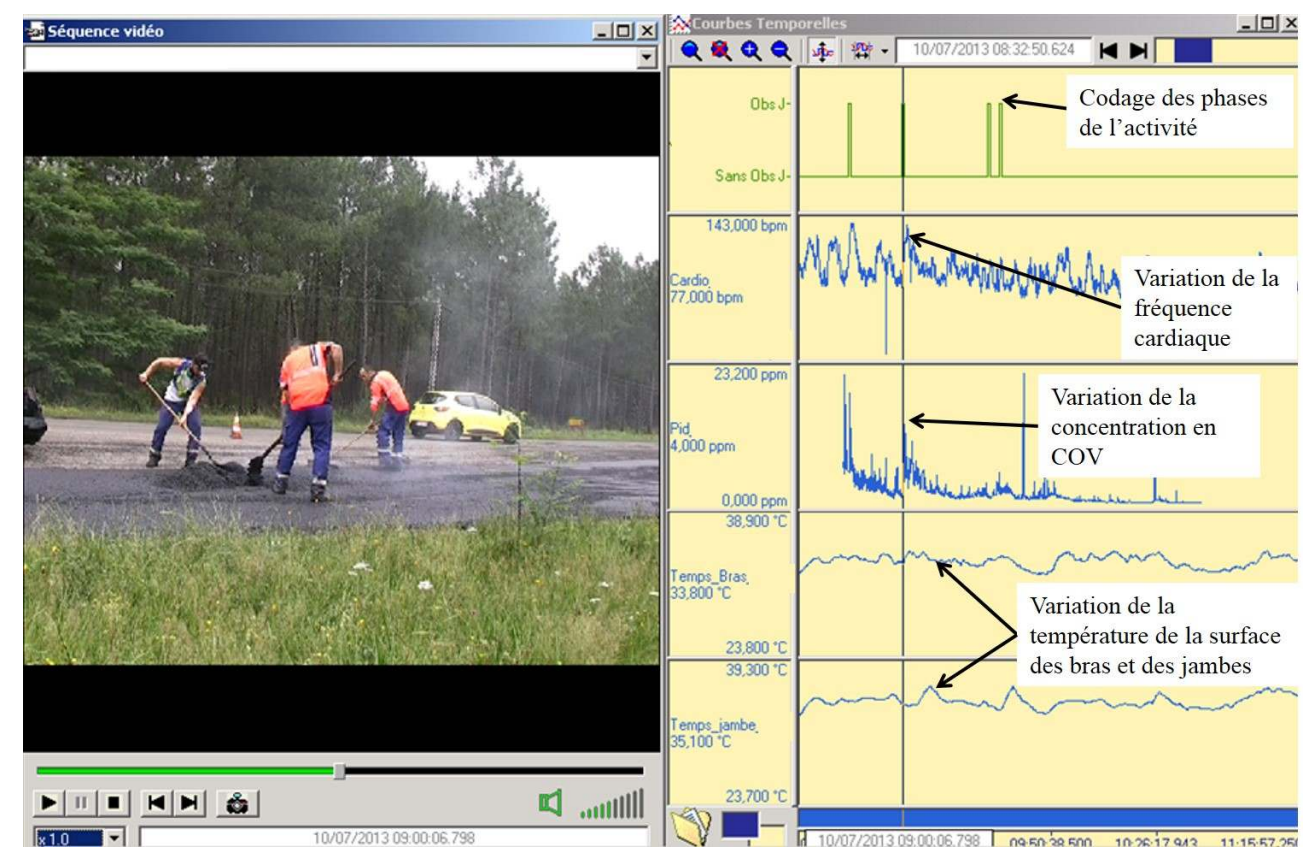

La figure 5 décrit une exposition au styrène de l'opératrice en train d'ébuller une coque de navire dans l'entreprise de fabrication de bateaux. L'analyse montre que, selon la forme de la coque ou de la carène, l'opératrice doit se pencher en avant pour repérer visuellement des déformations de la surface indiquant la présence de bulles et développer une gestuelle plus précise. Elle montre aussi que dans ces conditions, l'opératrice rapproche ses voies respiratoires du flux de vapeur de styrène qui se dégage de la coque. Des pics élevés de concentration (plus de 400 ppm) sont repérés et correspondent à la phase de nettoyage du pinceau et de la roulette avec de l'acétone. De plus, de nombreuses phases d'attente sont identifiées dans le déroulement de l'activité. Enfin, l'analyse de l'intensité physique correspond à une intensité modérée selon la grille de Meunier, Smolik et Knoché (1994). 
Figure 5 : Capture d'écran du logiciel Captiv ${ }^{\circledR}$ illustrant une tâche réalisée par une opératrice dans l'entreprise de fabrication de bateaux et les variations d'exposition associées.

Figure 5 : Captiv ${ }^{\circledR}$ L7000-2014 software screenshot, describing a task performed by the worker and the variation of exposure in a boat building company

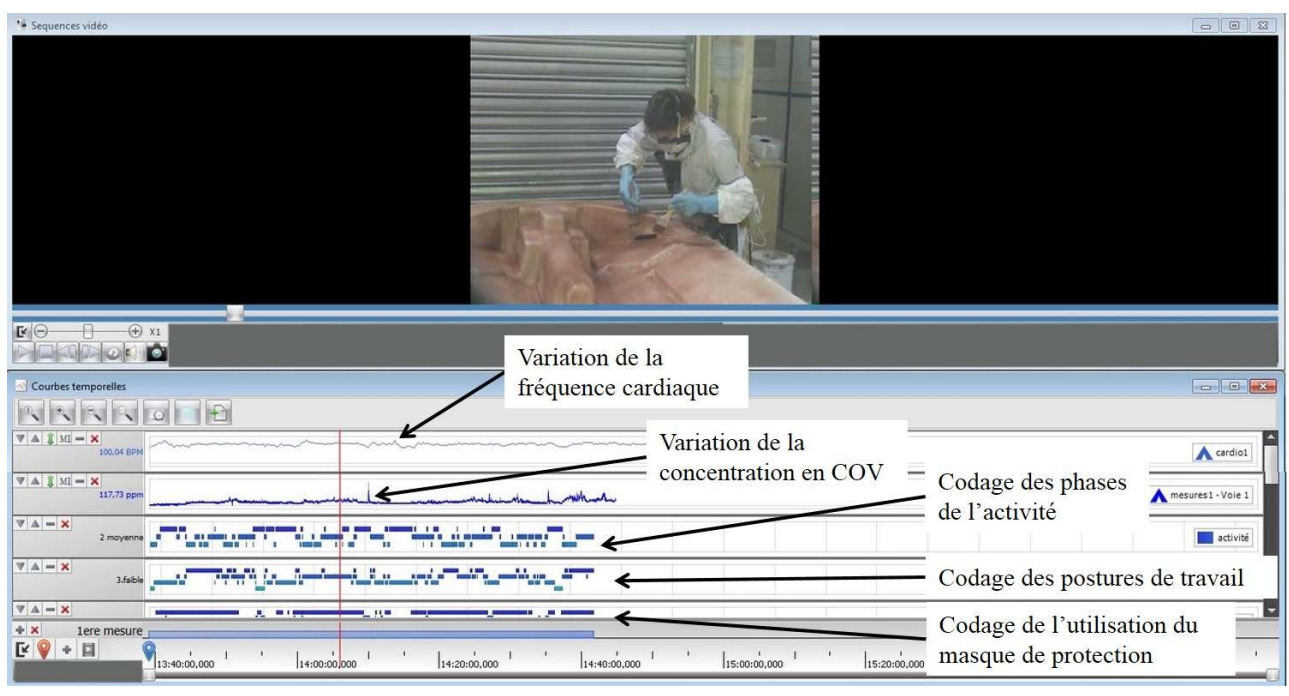

\subsection{Analyses statistiques issues de l'entreprise de fabrication de bateaux}

Dans cette partie, nous avons fait le choix de montrer ce que peut produire le traitement de données croisées, dans le cas de l'entreprise de fabrication de bateaux (étape 4 de la méthode, voir Figure 1). Premièrement, nous pouvons souligner que la tâche d'ébullage est composée de plusieurs phases. L'activité d'ébullage représente seulement $47 \%$ de la durée d'observation analysée; de longues périodes d'attente des opérateurs sont identifiées ( $24 \%$ de la durée totale de l'activité). Une analyse détaillée permet de mettre en avant que lorsque les opérateurs attendent l'arrivée d'une coque à ébuller, ils ont tendance à ne pas porter de masque bien qu'une exposition à des concentrations significatives de styrène soit mesurée. Cette exposition est liée aux vapeurs de styrène dégagées par les coques avoisinantes. Cette technique d'analyse permet également de mettre en avant que l'opérateur est exposé à une concentration supérieure à 50 ppm (la valeur limite d'exposition professionnelle au styrène avant 2018) pour $82 \%$ de la durée de l'ébullage, $29 \%$ des périodes d'attente, $84 \%$ de la durée du contrôle et $46 \%$ des périodes de manipulation (voir Figure 5, diagramme en haut à droite). Si par contre, on prend en compte la valeur d'exposition du styrène utilisée en Allemagne ou bien aux USA, qui est une valeur cible des recommandations faites par l'ANSES, elle se situe à $20 \mathrm{ppm}$. Le traitement croisé des données sur cette base fait apparaitre que cela correspond à $100 \%$ de la durée pour toutes les phases sauf celle d'attente qui se situe à $80 \%$ (voir Figure 5 , diagramme en haut à gauche). Cette projection permet d'attirer l'attention de l'entreprise sur les niveaux d'exposition sur le poste d'ébullage et sur la nécessité d'anticiper le changement règlementaire à venir. Enfin, il a été montré que le masque de protection respiratoire est porté pendant $87 \%$ de la durée de réalisation de la tâche. Si l'on s'intéresse au niveau de concentration auxquels est exposé la travailleuse lorsqu'elle ne porte pas de masque, le traitement croisé fait apparaître que pendant près de $18 \%$ de la phase d'attente elle est exposée à une concentration supérieure à 50 ppm (voir Figure 6, 
diagramme en bas à droite) et près de $28 \%$ à une concentration supérieure à 20 ppm (voir Figure 6, diagramme en bas à gauche).

Figure 6 : Statistiques croisées sur les variations d'exposition en fonction des phases de l'activité de travail dans l'entreprise de fabrication de bateaux.

Figure 6 : Crossed statistics of exposure variations depending on work activities in the boat building company
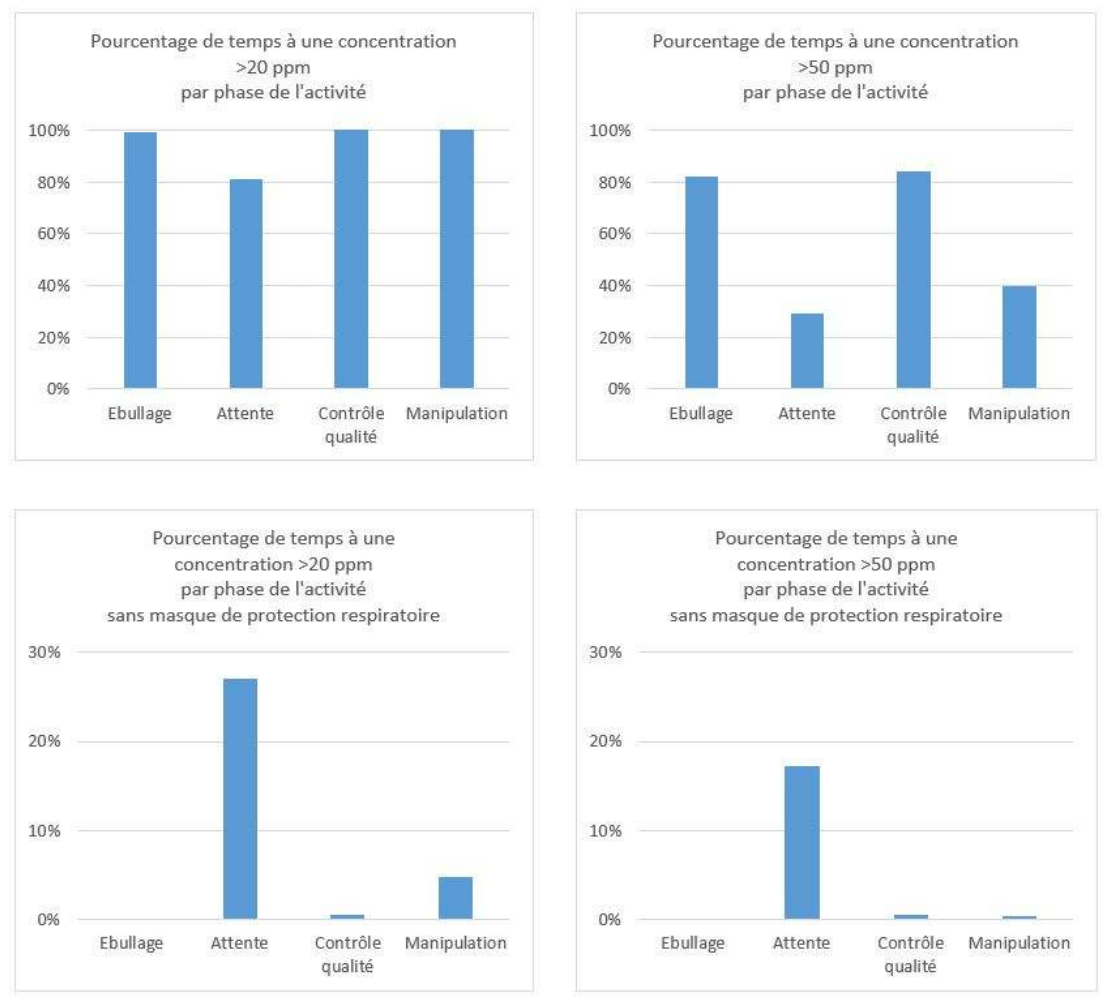

\subsection{Résultats issus des entretiens de confrontation}

Le tableau 2 présente en fonction de chaque entreprise des extraits d'échanges sur l'exposition issus d'entretiens de confrontation (étape 5 de la méthode, voir Figure 1). Pendant ces entretiens de confrontation, le visionnage simultané de l'activité synchronisée aux données de mesure permet d'identifier le moment et les conditions des expositions. Ce support de discussion aide les travailleurs à décrire et questionner ce qu'ils font en considérant les niveaux d'exposition associés. Les informations de contexte enregistrées par la vidéo ou l'observation parallèle alimentent ces entretiens de confrontation. Nous soutenons l'importance de cet usage de la VEM pour la prévention, grâce à la combinaison de données objectives (mesurage, description de l'activité de travail...) à des données subjectives (représentation et analyse collective de scénario d'exposition...) propre à la pratique de l'ergotoxicologie. Ce couplage contribue ainsi à mieux comprendre les expositions et surtout à les corréler avec certaines phases de l'activité de travail. Au niveau individuel, nous observons que les opérateurs, qui se voient exposés à des pics de concentration, découvrent leur exposition "par l'activité », une autre approche du risque chimique leur est dévoilée. Au niveau collectif, cela alimente une mise en débat des pratiques et peut mener à une évolution des représentations associées à des activités de travail. 
Tableau 2 : Verbatim résultants des entretiens de confrontation à partir de la VEM. Table2 : Verbatim resulting from confrontation interviews based on VEM

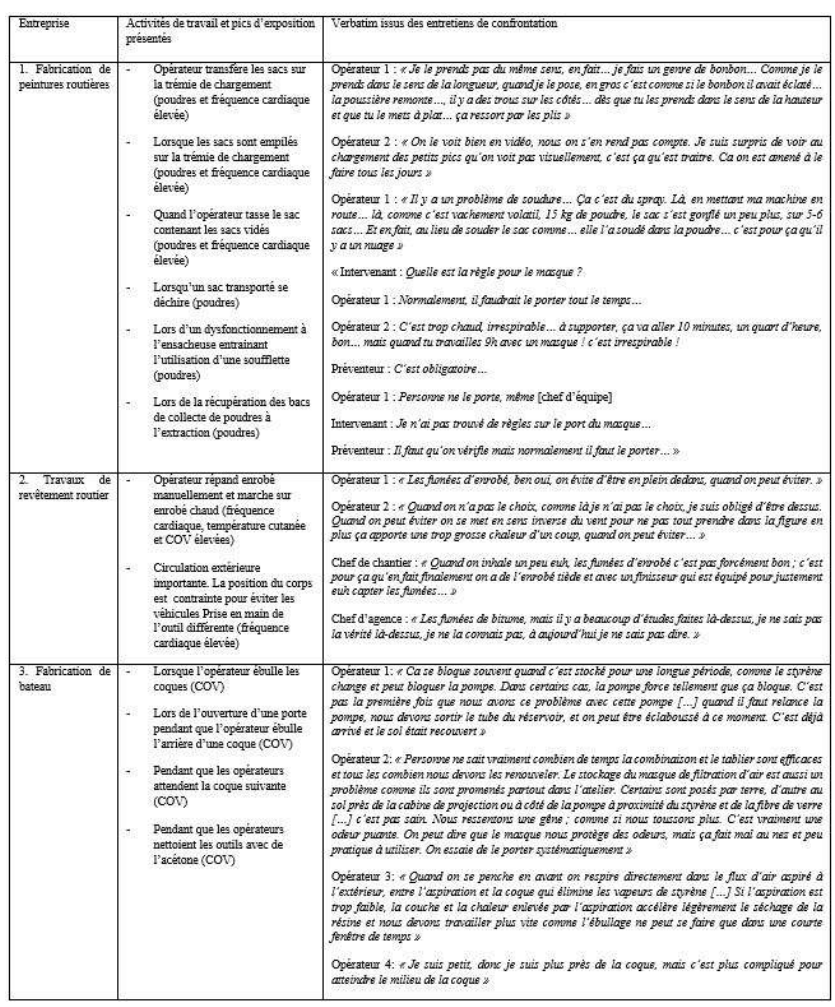

Dans l'entreprise de peinture routière, le document d'évaluation des risques comprend une seule situation d'exposition aux poussières (chargement des matières premières) alors que six situations sont révélées par la méthode. La description de ces situations d'exposition apportées par la VEM vient enrichir le document unique.

La mise en circulation de vidéo et mesures est un moyen de rendre visible l'exposition. Ces situations indétectables au préalable deviennent visibles à l'ensemble des acteurs de l'entreprise (opérateur et encadrement) auxquels sont présentés les résultats, renforçant ainsi les connaissances sur l'exposition. Par exemple, les aléas fréquents à l'ensacheuse gérés par les opérateurs n'apparaissent pas dans les pratiques formelles de gestion des risques et sont inconnus de certains membres de l'encadrement. Des facteurs organisationnels (réglage de l'ensacheuse par l'équipe de maintenance) expliquent ces aléas conduisant aux situations d'exposition : les opérateurs doivent manipuler plusieurs fois des sacs ouverts ou avec une quantité de poudre trop faible ou importante. L'utilisation de la soufflette en découle afin de souffler les poudres déposées dans l'ensacheuse ou sur les bandes de soudage de l'ensacheuse.

Les protections collectives peuvent être développées pour réduire la présence de poudres dans l'atmosphère de l'atelier (fuite au niveau du conduit de la ventilation et aspiration insuffisante à l'ensacheuse lors des aléas). De plus, des règles de sécurité non opérationnelles sont discutées comme le port d'un masque de protection respiratoire FFP3 sur l'ensemble du poste alors qu'une fréquence cardiaque élevée est enregistrée correspondant à un coût cardiaque lourd à très lourd (Meunier et al., 1994). La conjonction d'un effort cardiaque élevé qui entraîne une fréquence ventilatoire élevée et une exposition à une concentration élevée de particules conduit à identifier qu'un 
masque de type FFP3 n'offre pas une protection suffisante. Un flou de prescription autour du caractère obligatoire du port de ce masque est discuté collectivement lors d'un entretien de confrontation avec l'encadrement. À l'issue des entretiens de confrontation avec les acteurs de l'entreprise, de nouvelles actions de prévention élaborées lors des échanges, à partir de ces situations d'exposition, ont été validées en comité d'hygiène, de sécurité et des conditions de travail pour tendre vers une sécurité plus opérationnelle.

Dans l'entreprise de travaux routiers, les confrontations individuelles avec l'équipe, le chef d'agence et le préventeur montrent que les actions de prévention se focalisent sur une prévention formelle (Mohammed-Brahim, 1999): challenges - sécurité comptabilisant le nombre d'accidents du travail sur les douze mois glissants ou visites « sécurité » organisées régulièrement au sein de l'entreprise par le préventeur et/ou les conducteurs de travaux afin de contrôler le respect des consignes de sécurité. Ces actions visent à circonscrire les risques en régularisant les pratiques des opérateurs, considérés comme des cibles à atteindre et non pas comme des acteurs de leur prévention. La question de l'exposition cutanée au bitume n'est pas abordée spontanément, car ce risque est perçu comme incertain. L'état actuel des connaissances scientifiques sur l'exposition à des substances chimiques dans les travaux de revêtements routiers, du point de vue de la toxicologie et de l'épidémiologie, indique un contexte incertain renforcé par l'absence de tableau de maladies professionnelles spécifiques au bitume et de l'absence de valeur limite pour l'exposition à cette substance (Judon, 2017).

Ainsi, en mettant par exemple en visibilité, par l'intermédiaire de l'outil VEM, des coûts cardiaques lourds sur certaines phases de l'activité corrélés avec des concentrations plus importantes en $\mathrm{COV}$ recueillies à proximité des opérateurs, la question d'une possible augmentation de la pénétration cutanée a pu être mise en discussion avec l'ensemble des acteurs impliqués dans le processus (opérateurs, encadrement de proximité, préventeurs et décideurs au niveau national). La présentation des résultats de mesure sur les niveaux d'effort imposés par l'activité a amené les décideurs à prendre conscience du morcellement du processus d'analyse des risques au sein l'entreprise. En effet, un découpage par familles de risques y est réalisé, sans articulation entre chacune d'elles et sans prise en compte des conditions réelles de travail : la question de l'intensité physique du travail est alors déconnectée de celle de l'exposition au risque chimique, alors que l'augmentation de l'effort se traduit par une augmentation des volumes d'air inhalés et donc une augmentation de l'exposition aux substances chimiques.

Dans une perspective de recherche en prévention, les conditions d'un dialogue entre différents mondes professionnels au sein d'un même secteur d'activité ont été recherchées. L'émergence d'un « espace intermédiaire de dialogue » a été rendue possible par la co-construction d'objets intermédiaires. Cette co-construction s'est réalisée, à partir des supports de VEM, en capacité de produire et de soutenir un débat autour des pratiques effectives des opérateurs ainsi que de leurs représentations au regard des pratiques de sécurité mises en œuvre et appliquées par l'encadrement. Cet espace de dialogue a permis aux différents acteurs de s'investir et de mobiliser leurs ressources individuelles, collectives et organisationnelles pour apporter un autre regard sur les pratiques actuelles permettant d'imaginer de nouvelles pratiques de prévention dans un contexte incertain.

39 Dans le cadre de l'entreprise de fabrication de bateaux, l'outil de VEM a contribué à mettre en débat différents problèmes rencontrés par les travailleurs. Dans un premier temps, la mise en visibilité des périodes d'attente et des expositions associées a soulevé la 
question des modes dégradés de production et des actions de maintenance qui génèrent des retards que subissent les travailleurs. La caractérisation des expositions a donné l'occasion aux travailleurs de mettre en mots les gênes et les symptômes occasionnés par les expositions, en particulier des maux de tête et des maux de ventre. Il est à noter que ces éléments qui pourraient être classés comme subjectifs correspondent aux symptômes faisant suite à des expositions aigües dans les études toxicologiques.

Les questions liées à l'efficacité des moyens de protection collectifs comme individuels ont été largement discutées. Les aspirations seraient alors insuffisantes et accélèreraient la vitesse de séchage de la résine, ce qui exige alors d'ébuller plus rapidement avant que la surface ne durcisse. Concernant le port de masque, les opérateurs ont eux-mêmes identifié la nécessité de le porter lors des phases d'attente, ce qui peut entrer en contradiction avec le fait de devoir communiquer.

41 Malheureusement, il n'a pas été possible d'accompagner l'entreprise dans le déploiement des modifications, car celle-ci a été délocalisée en Espagne.

\section{Discussion}

42 L'utilisation de la vidéo est orientée vers le « dire », l'image aidant la parole du travailleur (Lacoste, 1995). L'image constitue en effet une aide pour que l'opérateur puisse mettre en mots son activité. Par définition, les productions verbales sur le travail sollicitées par l'intervenant ne sont pas spontanées. Les connaissances, les savoirs, les représentations mises en jeu pendant le travail sont difficilement verbalisables (Boutet, 1995, Judon, 2017 ; Odonne et al., 1981; Schwartz, 1989). L'usage d'un «support matériel d'évocation de l'action» (Béguin, 1997) est une condition minimale pour aider cette mise en mots. L'image facilite ainsi la production d'un discours sur le travail. Aussi, l'image, par les échanges qu'elle suscite avec le chercheur-intervenant, permet à l'opérateur de «se voir en activité ", et d'y découvrir des éléments nouveaux. La psychologie et l'ergonomie utilisent la confrontation à des enregistrements audio et vidéo des activités comme outil pour faciliter un retour réflexif sur l'activité en cours (Judon, 2017).

Notre usage de la VEM assure un espace réflexif (Hendry \& Seidl, 2003; Nascimento \& Mollo, 2014; Schön, 1983) où les opérateurs et l'encadrement peuvent développer une analyse discursive à partir des situations d'exposition. Cet usage permet une prise de conscience et un partage d'informations pertinentes et compréhensibles. Des éléments de contexte, comme l'environnement de travail, les autres dangers présents ou le contexte socio-organisationnel, conditionnant ces situations d'exposition peuvent être décrits et partagés par les acteurs mobilisés. Le travail des opérateurs devient accessible et est dévoilé à l'encadrement (Rocha, Mollo, \& Daniellou, 2015). En parallèle, la mesure renforce la mise en visibilité de polluants pas toujours perceptibles par les sens. Ainsi, la mesure renforce une objectivation de l'exposition déjà en partie apportée par l'activité. Cela permet une autre approche du risque chimique en partant de l'activité de travail, comme fil conducteur, pour remonter aux expositions (Garrigou \& Peissel-Cottenaz, 2008 ; Judon, 2017). L'exposition est abordée en lien avec le travail, permettant aux opérateurs d'en retrouver le sens et la compréhension, contrairement aux approches expertes portées par des acteurs extérieurs. Garrigou et al. (2015) et Galey, Barcellini, Rinaldo, et Garrigou (2014) renforcent ce constat en soulignant les possibilités d'agir des acteurs de la prévention (opérateurs compris) sur des facteurs qui déterminent l'exposition. Nos résultats montrent que la forme de présentation des situations 
d'expositions, permise par la VEM, autorise un dialogue sur l'activité de travail. Ainsi, la possibilité offerte aux opérateurs de verbaliser sur leur activité rendue visible (étape 5 de notre méthodologie) et sur les difficultés ou contraintes rencontrées dans la réalisation du travail (Garrigou, 2011) renforce la compréhension des actions qu'ils mettent en œuvre. À partir des connaissances développées par les opérateurs, comme des savoirfaire et des représentations des risques présents, des limites de pratiques de sécurité sont décelables et mises en débat avec l'encadrement. Il ne s'agit donc pas de convaincre, ni de révéler de mauvaises pratiques ou encore d'imposer de nouvelles bonnes pratiques, mais de permettre un apprentissage partagé (Béguin, 2014) des savoirs déjà mobilisés dans l'activité pour se protéger des expositions et autres dangers présents.

L'usage de ce type d'outil dévoilant en partie l'activité permet, par exemple, de mieux comprendre certaines prises de risque ou non-respect de consignes. Le regard des autres membres du collectif face à la résistance aux situations à risque va pouvoir être révélé (Garrigou, 2011). En effet, des stratégies défensives, comme le déni du risque, peuvent avoir été construites pour se convaincre de l'innocuité d'une situation; de nouvelles consignes peuvent, par exemple, paraître disproportionnées face à un danger perçu au départ comme inoffensif. Sur la base de la méthodologie présentée, il est alors possible de dégager de nouvelles perspectives de prévention, qui peuvent être proposées par les opérateurs à partir des savoirs issus de leur expérience.

La mobilisation de la VEM dans une méthodologie d'intervention va interroger les déterminants organisationnels, techniques ou humains des expositions. De plus, la compréhension des activités, des représentations et savoirs, et des expositions au cours du travail ouvre de réelles perspectives d'action pour des interventions et recherches en ergonomie. Enfin, l'implication des opérateurs ne doit pas se limiter à imaginer des solutions de prévention, il faut rester attentif à leur réelle participation au processus de transformation.

Il apparaît par ce travail que la mise en circulation de données de mesure et d'observation vidéo est une ressource pour un échange collectif. Dans le cas de l'entreprise de peinture routière, la règle autour de la prescription du port du masque est remise en question. Les opérateurs partagent avec l'encadrement la pratique développée de non-port de protection. Elle est expliquée par l'inconfort suscité par cette protection individuelle, inconfort associé à un travail d'intensité physique mesurée importante. Les échanges entre opérateurs et encadrement amènent à conclure à un flou de prescription sur le caractère obligatoire du port du masque, invisible dans les documents de prévention de l'entreprise. Les opérateurs défendent le point de vue collectif de l'impossibilité d'appliquer cette règle potentielle quand le préventeur insiste sur la nécessité de se protéger au vu des résultats de mesure. Si ces premiers résultats ouvrent des perspectives, un travail reste à mener pour comprendre comment la mesure peut devenir un moyen de mettre en évidence des savoir-faire, développer des connaissances et échanges collectifs sur le travail (Caroly \& Barcellini, 2013) et l'exposition, ou encore défendre le point de vue d'acteurs de l'entreprise.

\subsection{Précautions méthodologiques}

47 Nous relevons cependant plusieurs précautions autour de l'usage de la mesure comme instrument d'intervention qui nous semblent importantes à prendre en compte. 

résultats. Des résultats de mesure faibles peuvent freiner la nécessité d'agir en prévention ou contribuer à développer une communication rassurante. La réalisation de mesure n'est pas une action de prévention en soi. L'affichage de ce type de collaboration doit ouvrir sur le développement effectif de la prévention sans se limiter à de la communication sur l'évaluation. Les résultats de mesure doivent toujours être situés dans leur contexte. La mesure produit une image de l'exposition à un moment donné dans une situation de travail particulière avec un opérateur à l'activité et aux représentations singulières. La réalisation de mesures nécessite d'identifier le contexte dans lequel émane la demande de l'entreprise: réponse à des attentes règlementaires, opportunité, développement d'activité, besoin réel et volonté. La mesure doit permettre de répondre à un besoin de l'entreprise afin d'optimiser les chances d'agir sur les situations d'exposition. Ce besoin est à identifier par les intervenants ou chercheurs en collaboration avec les acteurs de l'entreprise, dès le début de la structuration de la méthode. L'impact de l'intervention est renforcé par la maturité des entreprises en termes de prévention. Les formes de restitution, ressources et supports laissés à l'entreprise pour agir de manière autonome se posent.

51 En fonction du principe de fonctionnement des instruments de mesure en temps réel, seules certaines caractéristiques $d u$ polluant vont être décrites. Des interférences peuvent se produire si d'autres sources de polluants similaires au polluant d'intérêt sont présentes dans l'air ambiant pouvant fausser la détection. Aussi, l'absence de variations enregistrées peut entraîner une sous-estimation de la présence de polluants.

Les questions éthiques (propriété de l'image, modalités de restitution, etc.) doivent faire l'objet d'un accord, en amont, avec les acteurs de l'entreprise. Dans notre pratique, la présentation des résultats de mesures et observations vidéo aux opérateurs avant l'encadrement est une manière supplémentaire de valider la possibilité d'utiliser ultérieurement ces supports.

53 Implémenter ce type de stratégie nécessite des connaissances en hygiène industrielle afin d'identifier, par exemple, le type de substances dangereuses présent dans l'environnement de travail, les propriétés physico-chimiques associées, le travail réalisé, les possibles voies de pénétration de ces substances dans l'organisme, ou encore les 
relations dose effet, etc. Ces connaissances vont alors guider l'observation de l'activité et plus particulièrement les caractéristiques de ces situations d'exposition pouvant être déterminées par des facteurs techniques, humains ou organisationnels. À son tour, l'observation ergonomique de l'activité de travail peut contribuer à améliorer la stratégie de mesure initiale. La méthode construite avec l'entreprise doit rendre possible l'accès aux variabilités et stratégies de régulation des opérateurs. Les entretiens d'auto confrontation et de confrontation collective menés autorisent les travailleurs à verbaliser sur leurs savoir-faire.

\subsection{La VEM pour la pluridisciplinarité et les échanges transprofessionnels}

$54 \mathrm{Au}$ sein des trois recherches-interventions présentées, des collaborations avec des disciplines différentes ont été initiées. Un travail commun avec des chimistes, métrologues, toxicologues a été mené afin de préciser, dans chaque cas, les mesures à réaliser et leur interprétation. La VEM devient alors un objet intermédiaire au sens de Vinck (2009 et 2012) proposant une forme particulière de présentation de l'activité qui invite à la pluridisciplinarité. En effet, cette approche propose plusieurs points d'entrée aux professionnels de diverses disciplines : la fréquence cardiaque pour les médecins du travail, la concentration en polluant pour les hygiénistes, la vidéo de l'activité et son codage pour les ergonomes. La présentation de l'activité devient alors un fil conducteur qui guide les acteurs dans leur compréhension de l'exposition. La mesure questionne l'activité réalisée en parallèle (Kloetzer, Quillerou-Grivot, \& Simonet, 2015). L'utilisation de données d'exposition synchronisées va conduire à caractériser irréfutablement l'activité et déterminer les situations au cours desquelles les opérateurs sont exposés à des dangers. L'approche par l'activité renforce la compréhension des interactions entre plusieurs facteurs de risque (polluants multiples, lien entre intensité physique de l'activité et expositions). De plus, l'utilisation de la mesure peut rassembler et coordonner différents points de vue pouvant être mobilisés dans l'analyse de la situation de travail, à condition qu'il y ait eu un accord avec les acteurs interagissant autour de ces situations (les opérateurs, mais également l'encadrement). Ainsi, une interprétation en collaboration avec les différents participants apporte davantage de poids pour convaincre les acteurs pouvant agir sur les situations de travail de changer ces situations. L'utilisation de la VEM avec les opérateurs uniquement aide à comprendre les situations d'exposition, comme les représentations et les raisonnements cognitifs, ce qui est nécessaire à la construction de manières appropriées de réduire l'exposition. La coconstruction de l'intervention favorise alors la production de connaissances sur le travail (Oddone, 1984) et les situations d'exposition. Une fois que les facteurs supposés influencer l'exposition sont identifiés, une stratégie pour caractériser l'ensemble des paramètres associés est à développer. Ces paramètres seraient habituellement mesurés séparément par les spécialistes de différentes disciplines. La VEM comme objet médiateur des échanges, entre les différents acteurs et disciplines de santé au travail rappelle la sélection d'objets-frontières ${ }^{1}$ dans les processus d'ergonomie participative. Ces objets sont essentiels pour favoriser la participation et l'implication des acteurs, donc une conception collaborative. Ils sont nécessaires pour créer un langage commun qui permet aux connaissances et aux idées de tous les participants d'être représentés (Broberg, Andersen, \& Seim, 2011). 

l'encadrement. En effet, les médecins du travail et les préventeurs participent rarement au processus de conception des situations de travail ou des procédés (Barcellini, Van Belleghem, \& Daniellou, 2014). En revanche, la décision et l'action de transformation dépendent fortement des acteurs et de leur accès aux logiques de projet. La méthodologie que nous proposons mobilise plusieurs acteurs impliqués dans la situation de travail. Ces espaces de discussion et de débat sont importants pour intégrer l'ensemble des points de vue et débattre collectivement des actions à construire (Rocha et al., 2015; Bolis \& Sznelwar, 2016). en ergonomie et dans d'autres champs où des mesures objectives peuvent être synchronisées à la vidéo (par exemple, l'exposition au bruit, l'exposition aux vibrations, l'étude des expositions environnementales...).

\section{Conclusion}

57 À travers ces trois cas décrits, nous montrons que des pratiques de l'hygiène industrielle et de l'ergonomie peuvent alimenter réciproquement les recherches et interventions. La mesure, les connaissances de modes d'action des substances grâce à l'hygiène industrielle et des méthodes de co-construction des interventions ou techniques d'entretien de l'ergonomie sont des ressources mutuelles.

La VEM facilite et encourage une implication significative des travailleurs dans le processus de prévention. Nous souhaitons également souligner que cette implication doit faire l'objet d'un accord préalable avec l'encadrement. Quand cela est possible, l'implication des opérateurs dans le processus de prévention permet de développer et de favoriser la conscience des situations exposantes à une contamination potentielle. Cette mise en visibilité ne concerne pas uniquement les opérateurs de terrain, mais également l'encadrement et les préventeurs. La VEM est ainsi un objet intermédiaire qui fournit à l'ensemble des acteurs une nouvelle perspective sur l'activité de travail et l'exposition. Elle révèle des situations souvent invisibles et crée la possibilité de partager des connaissances sur les situations de travail, les expositions, les questions autour du port des équipements de protections individuelles, mais aussi les questions concernant la production, son planning et son organisation, comme les pratiques de prévention et les savoir-faire développés par les opérateurs. La VEM n'est plus un objet de communication, mais s'intègre à une méthodologie pour développer les connaissances, pour comprendre et décrire les représentations et savoir-faire liés aux expositions, et possiblement les développer. Il s'agit bien d'aider à formuler l'énigme que constituent l'exposition et ses déterminants.

De notre expérience, il apparaît que les approches pluridisciplinaires sont par essence à géométrie variable. Plusieurs caractéristiques clés sont identifiées. La première est que la démarche soit collectivement construite. En d'autres termes, des hypothèses sont faites collectivement, la stratégie d'observation et de mesure ainsi que le protocole correspondant sont élaborés en collaboration, proposés par la discipline ou le professionnel le plus approprié pour générer une information pertinente.

60 Au-delà des opérateurs et de l'encadrement, la VEM est un outil au fort potentiel pour entraîner une coopération entre des professionnels de la prévention de différentes 
origines: médecins du travail, toxicologues, hygiénistes industriels, préventeurs ou ergonomes. Cela peut conduire à dépasser les approches « classiques» de la prévention en proposant une approche guidée par l'activité de travail, comme celle proposée par l'approche ergotoxicologique (Mohammed-Brahim, 2006; Mohammed-Brahim \& Garrigou, 2009).

Les développements récents en ergotoxicologie mobilisent des travaux qui portent sur les différentes formes de confrontations des travailleurs à leur activité (Clot, 2008; Clot, Faïta, Fernandez, \& Scheller, 2000 ; Mollo \& Falzon, 2004 ; Nascimento \& Mollo, 2014 ; Oddone et al. 1981) afin de pouvoir articuler des approches objectives et subjectives à différents niveaux d'acteurs. Les apports présentés dans cet article s'inscrivent dans la continuité de travaux précurseurs en ergotoxicologie (Sznelwar, 1992; Villate, 1985; Wisner, 1997). Révéler l'activité et les situations d'exposition est une condition au développement de l'activité et de la prévention des expositions professionnelles pour les femmes et les hommes au travail.

\section{BIBLIOGRAPHIE}

Barcellini, F., Van Belleghem, L., \& Daniellou, F. (2014). Design projects as opportunities for the development of activities. In P. Falzon (Éd.), Constructive Ergonomics (p. 187-204). CRC Press. https://doi.org/10.1201/b17456-16

Bardin, L. (2011). L'analyse de contenu (3e éd.). Paris : Presses Universitaires de France.

Béguin, P. (1997). Le caméscope, l'image et le mot. Quelques remarques sur l'usage de la vidéo pour l'analyse du travail. Champs visuels, 6, 61-67.

Béguin, P. (2014). The design of instruments viewed as a dialogical process of mutual learning. In P. Falzon (Ed.), Constructive Ergonomics (p. 143-156). CRC Press.

Beurskens-Comuth, P. A. W. V., Verbist, K., \& Brouwer, D. (2011). Video Exposure Monitoring as Part of a Strategy to Assess Exposure to Nanoparticles. Annals of Occupational Hygiene, 55(8), 937945. https://doi.org/10.1093/annhyg/mer060

Bolis, I., \& Sznelwar, L. I. (2016). A case study of the implementation of an ergonomics improvement committee in a Brazilian hospital - Challenges and benefits. Applied Ergonomics, 53, 181-189. https://doi.org/10.1016/j.apergo.2015.09.012

Boutet, J. (1995). Le travail et son dire. In J. Boutet (Ed.), Paroles au Travail (p. 247-267). Paris : L'Harmattan, Collection Langage et Travail.

Broberg, O., Andersen, V., \& Seim, R. (2011). Participatory ergonomics in design processes: The role of boundary objects. Applied Ergonomics, 42(3), 464-472. https://doi.org/10.1016/ j.apergo.2010.09.006

Clot, Y. (2008). Travail et pouvoir d'agir. 296 p. Paris, PUF.

Clot, Y. (2010). Au-delà de l'hygiénisme : l'activité délibérée. Nouvelle Revue de Psychosociologie, 10 (2), 41-50. 
Clot, Y., Faïta, D., Fernandez, G. \& Scheller, L. (2000). Entretiens en auto confrontation croisée : une méthode en clinique de l'activité. Perspectives interdisciplinaires sur le travail et la santé, 2(1). DOI : 10.4000/pistes.3833 http://journals.openedition.org/pistes/3833

Caroly, S., \& Barcellini, F. (2013). Le développement de l'activité collective. Ergonomie constructive (p. 33-46). Presses Universitaires de France.

Dagiral, É., Jouzel, J.-N., Mias, A., \& Peerbaye, A. (2016). Mesurer pour prévenir ? Terrains \& travaux, 28, 5-20.

Daniellou, F. (1992). Le statut de la pratique et des connaissances dans l'intervention ergonomique de conception. Habilitation à diriger des recherches, Université Victor Segalen-Bordeaux 2-ISPED, Laboratoire d'ergonomie des systèmes complexes.

Falzon, P. (2004). Ergonomie. Paris : Presses Universitaires de France.

Galey, L., Barcellini, F., Rinaldo, M., \& Garrigou, A. (2014). Exposition aux nanotubes de carbone dans une plateforme de transfert de technologies. In ACE 2014 Annual Conference Proceedings. Montréal, Canada.

Garrigou, A. (2011). Le développement de l'ergotoxicologie : une contribution de l'ergonomie à la santé au travail. Mémoire d'Habilitation à Diriger des Recherches en Ergonomie, Université de Bordeaux.

Garrigou, A., Carballeda, G., \& Daniellou, F. (1998). The role of 'know-how' in maintenance activities and reliability in a high-risk process control plant. Applied Ergonomics, 29(2), 127-131. https://doi.org/10.1016/S0003-6870(96)00060-9

Garrigou, A., Daniellou, F., Carballeda, G., \& Ruaud, S. (1995). Activity analysis in participatory design and analysis of participatory design activity. International Journal of Industrial Ergonomics, 15 (5), 311-327. https://doi.org/10.1016/0169-8141(94)00079-I

Garrigou, A., Peeters, S., Jackson, M., Sagory, P., \& Carballeda, G. (2004). 30. Apports de l'ergonomie à la prévention des risques professionnels. In P. Falzon, Ergonomie (1r éd., p. 497). Presses Universitaires de France. Consulté à l'adresse http://www.cairn.info/ ergonomie--9782130514046-page-497.htm

Garrigou, A., \& Peissel-Cottenaz, G. (2008). Reflexive approach to the activity of preventionists and their training needs: Results of a French study. Safety Science, 46(8), 1271-1288. https:// doi.org/10.1016/j.ssci.2007.07.006

Garrigou, A., Théry, L., Chassaing, K., Effantin, E., Mercieca, P., Dimerman, S., Vanderghote, M., Négroni, P., Gauthier, P., Goutille, F., Galey, L., Rambaud, C., Laporte, E., Merlin, X., Vergneaux, L., \& Baratta, R. (2015). Une approche pluridisciplinaire du processus de construction sociale de la prévention du risque CMR. In Actes du $50^{e}$ Congrès International de la Société d'Ergonomie de Langue Française (p. 476-489). Paris, France.

Hale, A., \& Borys, D. (2013). Working to rule or working safely? Part 2: The management of safety rules and procedures. Safety Science, 55, 222-231. https://doi.org/10.1016/j.ssci.2012.05.013

Hendry, J., \& Seidl, D. (2003). The Structure and Significance of Strategic Episodes: Social Systems Theory and the Routine Practices of Strategic Change. Journal of Management Studies, 40(1), 175196. https://doi.org/10.1111/1467-6486.00008

Horwat, F., \& Meyer, J. P. (1998). Débit ventilatoire de travail. Médecin du Travail, 76(4), 343-357. Judon, N. (2017). Rendre possible un espace intermédiaire de dialogue pour co-construire de nouvelles solutions de prévention dans un contexte d'incertitude. Cas des travaux de revêtements routiers. Thèse en ergonomie, Université de Bordeaux. 
Judon, N., Hella, F., Pasquereau, P., \& Garrigou, A. (2015). Vers une prévention intégrée du risque chimique lié à l'exposition cutanée au bitume des travailleurs de la route. Élaboration d'une méthodologie dans le cadre de l'ergotoxicologie. Perspectives interdisciplinaires sur le travail et la santé, 17(2). https://doi.org/10.4000/pistes.4586

Kerguelen, A. (1997). The Kronos Software: a tool for work activity analysis. Proceedings of 13th Triennial Congress of International Ergonomics Association, Volume 7, 240-247, Tampere, Finland, 29 juin-4 juillet.

Kerguelen A. (1998). Quels outils concevoir pour aider au relevé d'observation sur le terrain? XXXIIIème Congrès de la SELF, “Temps et Travail”, p. 870-813, Paris.

Kerguelen, A. (2003). Actogram Kronos pour Windows. Toulouse : Éditions Octarès.

Kloetzer, L., Quillerou-Grivot, E., \& Simonet, P. (2015). Engaging workers in WRMSD prevention: Two interdisciplinary case studies in an activity clinic. Work, 51(2), 161-173. https:// doi.org/10.3233/WOR-141970

Kuhl, K., \& Dobernowsky, M. (2011). Application of PIMEX method: employees are motivated to change their working conditions and optimise preventive measures. Work (Reading, Mass.), 39(4), 379-384. https://doi.org/10.3233/WOR-2011-1188

Lacoste, M. (1995). Parole, activité, situation. In J. Boutet (Ed.), Paroles au travail (p. 23-44). Paris : L'Harmattan.

Martin, P., Brand, F., \& Servais, M. (1999). Correlation of the Exposure to a Pollutant with a TaskRelated Action or Workplace : The CAPTIVTM System. The Annals of Occupational Hygiene, 43(4), 221-233. https://doi.org/10.1016/S0003-4878(99)00026-5

Meunier, P., Smolik, H.J., Knoché, C. (1994). Astreinte cardiaque et travail. Quelle grille d'évaluation choisir? Cahiers de Médecine Interprofessionnelle CAMIP, p. 153-158.

Mias, A., Legrand, E., Carricaburu, D., Féliu, F., \& Jamet, L. (2013). Le travail de prévention. Les relations professionnelles face aux risques cancérogènes. Toulouse : Éditions Octarès.

Mohammed-Brahim, B. (1999). Ambiances chimiques de travail : l'ergotoxicologie ou la transition d'une prévention formelle à une prévention opérationnelle. Performances Humaines et Techniques, no 99, 27-34.

Mohammed-Brahim, B. (2006). Concept and methods in ergotoxicology. In International Encyclopedia of Ergonomics and Human Factors (Taylor \& Francis, vol. 1, p. 698-705). UK : W. Karwowski.

Mohammed-Brahim, B., \& Garrigou, A. (2009). Une approche critique du modèle dominant de prévention du risque chimique. Activités, 6(1). https://doi.org/10.4000/activites.2086

Mollo, V., \& Falzon, P. (2004). Auto- and allo-confrontation as tools for reflective activities. Applied Ergonomics, 35(6), 531-540. https://doi.org/10.1016/j.apergo.2004.06.003

Mollo, V., \& Nascimento, A. (2014). Reflective practices and the development of individuals, collectives and organizations. In P. Falzon (Ed.), Constructive Ergonomics (p. 236-251). CRC Press.

Monod, H., \& Pottier, M. (1981). Les adaptations respiratoires et circulatoires du travail musculaire. In J. Scherrer (Ed.), Précis de physiologie du travail. Notions d'ergonomie (p. 292-327). Paris : Masson.

Oddone, I., Re, A., Briante, G. (1981). Redécouvrir l'expérience ouvrière, vers une autre psychologie du travail ? Paris : Éditions Sociales.

Oddone, I. (1984). La communauté scientifique élargie. Revue Société Française, 10. 
Rocha, R., Mollo, V., \& Daniellou, F. (2015). Work debate spaces: A tool for developing a participatory safety management. Applied Ergonomics, 46, 107-114. https://doi.org/10.1016/

j.apergo.2014.07.012

Rosén, G., Andersson, I.-M., Walsh, P. T., Clark, R. D. R., Säämänen, A., Heinonen, K., ... Pääkkönen, R. (2005). A Review of Video Exposure Monitoring as an Occupational Hygiene Tool. Annals of Occupational Hygiene, 49(3), 201-217. https://doi.org/10.1093/annhyg/meh110

Schwartz, Y. (1989). « C'est compliqué » : activité symbolique et activité industrieuse. Langage, 93, 98-109.

Schön, D. A. (1983). The reflective practitioner. How professionals think in action. New York : Basic Books. Traduction française : Le praticien réflexif. À la recherche du savoir caché dans l'agir professionnel, 1994. Éditions Logiques, Montréal.

Star, S.L. (2010). Ceci n'est pas un objet-frontière : Réflexions sur l'origine d'un concept. Revue d'Anthropologie des Connaissances, 4(1), 18-35.

Sznelwar, L. (1992). Analyse ergonomique de l'exposition de travailleurs agricoles aux pesticides : Essai ergotoxicologique. Thèse de doctorat en ergonomie. Laboratoire d'ergonomie du CNAM, Paris.

Trompette, P., \& Vinck, D. (2009). Retour sur la notion d'objet-frontière. Revue d'Anthropologie des Connaissances, 3(1), 5-27.

Vidal-Gomel, C. (2017). Training to safety rules use. Some reflections on a case study. Safety Science, 93(Supplement C), 134-142. https://doi.org/10.1016/j.ssci.2016.12.001

Villate, R. (1985). Toxicologie et ergonomie. In B. Cassou, D., Huez, M.-L., Mousel, C., Spitzer, A., Touranchet-Hébrard, (Eds.), Les risques du travail (p. 301-303). Paris : La Découverte.

Vinck, D. (2009). From Intermediary Object towards Boundary-Object. Revue d'anthropologie des connaissances, 3(1), 51-72.

Vinck, D. (2012). Accessing Material Culture by Following Intermediary Objects. In An Ethnography of Global Landscapes and Corridors. InTech.

Wisner, A. (1972[1995]). Le diagnostic en ergonomie ou le choix des modèles opérants. In A. Wisner (1995), Réflexions sur l'ergonomie (1962-1995) (p. 79-102). Toulouse : Octarès.

Wisner A. (1997). Ergotoxicologie dans les pays tropicaux. In A. Wisner (Ed.), Anthropotechnologie, vers un monde industriel pluri centrique, $1^{\mathrm{e}}$ édition (p. 179-189). Toulouse : Octarès.

\section{NOTES}

1. Les acteurs des différents mondes sociaux peuvent, grâce à l'objet-frontière, maximiser leur communication « inter » mondes, négocier leurs différences et créer un accord entre leurs points de vue respectifs (Trompette \& Vinck, 2009). Il s'agit au-delà de s'accorder sur l'existence de « mondes » hétérogènes, de prendre en compte leur articulation et les mécanismes d'intersection de ces mondes dans une activité coordonnée (Star, 2010). 


\section{RÉSUMÉS}

L'ergotoxicologie a constitué depuis une quinzaine d'années un corpus conceptuel et méthodologique (Garrigou, 2011; Mohammed-Brahim \& Garrigou, 2009) afin de contribuer à la prévention des expositions des travailleurs aux produits chimiques. Faisant suite au texte de Mohammed-Brahim et Garrigou (2009) publié dans cette revue, cet article présente les derniers développements méthodologiques en ergotoxicologie et en particulier les apports de l'usage de mesures en temps réel synchronisées à des enregistrements vidéo de situations de travail. La méthodologie fait alors appel à une technique de video exposure monitoring (VEM), dont l'objectif est de "révéler " des situations d'exposition à des produits chimiques, afin de les mettre en débat au sein des entreprises et les transformer dans une logique préventive. Cette contribution méthodologique s'appuie sur un retour d'expérience basé sur trois études de cas. Enfin, nous discutons les perspectives de cette méthodologie pour l'ergonomie afin de développer des pratiques de prévention impliquant les travailleurs, leur encadrement et les acteurs de la santé au travail.

Over the last fifteen years ergotoxicology has been using an interdisciplinary approach to develop models and methods (Mohammed-Brahim \& Garrigou, 2009; Garrigou, 2011) in an effort to preserve occupational health. This approach is based on toxicological knowledge and on methods of work activity analysis. The purpose of this article is to present a method based on the use of real time measurements, synchronized with video recordings of work situations. This stems from methodological development in ergotoxicology. The methodology calls for use of the video exposure monitoring (VEM) technique to detect situations of exposure and to formulate an in-depth work activity analysis. The objective of this method is to uncover situations where there is chemical exposure and to help companies recognize this. Three case studies are presented in this article. In the conclusion, we discuss the perspectives of this methodology for ergonomics with a view to to developing prevention practices involving workers, their management and occupational health enablers.

\section{INDEX}

Keywords : video exposure monitoring, measurement, work activity analysis, ergotoxicology, exposure assessment

Mots-clés : analyse vidéo de l'exposition, mesure, analyse de l'activité, ergotoxicologie, évaluation des expositions

\section{AUTEURS}

\section{LOUIS GALEY}

Univ. Bordeaux, INSERM, Bordeaux Population Health Research Center, team EPICENE, UMR 1219, 146 rue Léo Saignat, 33000 Bordeaux, France.

louis.galey@u-bordeaux.fr 


\section{NATHALIE JUDON}

Univ. Bordeaux, INSERM, Bordeaux Population Health Research Center, team EPICENE, UMR 1219, 146 rue Léo Saignat, 33000 Bordeaux, France.

Département Homme au Travail, INRS, Rue du Morvan, CS 6002754519 Vandœuvre-lès-Nancy, France.

nathalie.judon@inrs.fr

\section{CAROLINE JOLLY}

Direction de la recherche et de l'expertise, Institut de recherche Robert-Sauvé en santé et en sécurité du travail, 505 Boulevard de Maisonneuve Ouest, Montréal, QC H3A 3C2, Canada. caroline.jolly@irsst.qc.ca

\section{FABIENNE GOUTILLE}

Univ. Bordeaux, INSERM, Bordeaux Population Health Research Center, team EPICENE, UMR 1219, 146 rue Léo Saignat, 33000 Bordeaux, France.

fabienne.goutille@u-bordeaux.fr

\section{SARAH MORELOT}

Univ. Bordeaux, INSERM, Bordeaux Population Health Research Center, team EPICENE, UMR 1219, 146 rue Léo Saignat, 33000 Bordeaux, France.

sarah.morelot@u-bordeaux.fr

\section{MARION ALBERT}

Univ. Bordeaux, INSERM, Bordeaux Population Health Research Center, team EPICENE, UMR 1219, 146 rue Léo Saignat, 33000 Bordeaux, France.

marion.albert@u-bordeaux.fr

\section{OLIVIER LHOSPITAL}

CHU de Bordeaux - Service de Médecine du Travail et de Pathologie Professionnelle - Centre Hospitalier Pellegrin - Place Amélie Raba-Léon - PQR 2 - 33076 Bordeaux.

olivier.lhospital@chu-bordeaux.fr

\section{PATRICK MARTIN}

Mission Technologies Nouvelles, Département Ingénierie des Procédés, INRS, Rue du Morvan, CS 6002754519 Vandœuvre-lès-Nancy, France.

patrick.martin@inrs.fr

\section{CATHERINE NOEL-SUBERVILLE}

IUT of Bordeaux, Département Hygiène, Sécurité et Environnement, 15 rue de Naudet, 33175 Gradignan, France.

catherine.noel-suberville@u-bordeaux.fr

Univ. Bordeaux, Laboratoire NutriNeuro, UMR INRA 1286, Bâtiment UFR Pharmacie - 2eme Tranche - $2^{\mathrm{e}}$ Etage - CC34, 146 rue Léo Saignat, 33076 Bordeaux, France. 


\section{PIERRICK PASQUEREAU}

IUT of Bordeaux, Département Hygiène, Sécurité et Environnement, 15 rue de Naudet, 33175 Gradignan, France.

pierrick.pasquereau@u-bordeaux.fr

\section{AGNÈS AUBLET-CUVELIER}

Département Homme au Travail, INRS, Rue du Morvan, CS 6002754519 Vandœuvre-lès-Nancy, France.

agnes.aublet-cuvelier@inrs.fr ;

\section{BRAHIM MOHAMMED-BRAHIM}

ASTIA Toulouse, 9 rue du Docteur Delherm, 31100 Toulouse. mb.brahim@astia.fr.

\section{ALAIN GARRIGOU}

Univ. Bordeaux, INSERM, Bordeaux Population Health Research Center, team EPICENE, UMR 1219, 146 rue Léo Saignat, 33000 Bordeaux, France.

alain.garrigou@u-bordeaux.fr 